\title{
Significance of Spe rm Characteristics in the Evaluation of Male Infertility in a Tertiary Care Centre
}

\author{
Asif Baliyan*, Harshi Dhingra and Anita Tahlan \\ Dept. of Pathology, Government Medical College and Hospital, Chandigarh, India
}

\begin{abstract}
Background: Infertility is both a clinical and a public problem. Standard semen analysis is the surrogate measure of male fertility in clinical practice to determine prevalence of low sperm count including oligozoospermia and azoospermia and to assess the pattern and distribution of abnormal semen parameters in infertile men.

Methods: The retrospective study was conducted with compiling of the data from archival record over a period of three years from June 2013 to June 2016. A total of 933 male partners of women attending the fertility clinic of hospital between the ages of 20 and 50 years were recruited. The samples taken were primary infertility cases using simple random sampling technique. Semen analysis was performed according to the standards outlined by the World Health Organization (5th edition 2010). Parameters outlined included: Appearance, Volume, $\mathrm{pH}$, Sperm concentration, Motility, Morphology, Viability and White cell count.
\end{abstract}

Result: Out of 933 samples, normozoospermia was observed in 659 (70.6\%) males, oligozoospermia 170 (18.2\%), and azoospermia $104(11.1 \%)$. The azoospermic and oligozoospermic samples had low ejaculated volume, but significantly higher percentage of pus cells in comparison to normozoospermic samples. The oligozoospermic samples had higher percentage of immotile sperms and abnormal morphology in comparison to normozoospermic samples. Asthenozoospermia was observed in $118(14.2 \%)$, teratozoospermia in 24 ( $2.9 \%)$, and oligoteratozoospermia in $11(1.3 \%)$ of samples.

Conclusion: Majority of cases of infertility in males show normal sperm count. Oligozoospermia followed by azoospermia is seen in rest of the cases while less sperm motility or less amount of semen are also responsible in some cases.

Keywords: Semen Analysis, Male Infertility, Sperm Motility, Morphology, Azoospermia.

\section{Introduction}

Infertility is a comprehensive issue affecting approximately $13-15 \%$ of the population all over the world. ${ }^{[1]} \mathrm{A}$ host of factors have been implicated in the causation of infertility. ${ }^{[1]}$ The female factor is responsible for $35 \%$ of cases whereas the male factor is seen in $45 \%$ of cases. The remaining $20 \%$ of the couples either have non-identified infertility or mixture of factors. ${ }^{[2]}$

The role of semen analysis in the assessment of male fertility is paramount and remains the most fundamental and primary investigation. ${ }^{[3]}$ The use of standardized and objective procedures ensures satisfactory categorization of cases of infertility. ${ }^{[3]}$ The results of the test provide vital information regarding the concentration, motility and morphology of the spermatozoa in a semen sample. ${ }^{[1]}$ The sensitivity value of standard semen analysis is $89.6 \%$, which implies that it has the ability to identify 9 out of 10 men having a genuine problem. ${ }^{[4]}$ Semen analysis allows for a better understanding of the structural and dynamical parameters involved in sperm function. ${ }^{[2]}$ The analysis also helps to elucidate the pathological causes for decreased sperm count thereby classifying the issue into a pretesticular, testicular or post testicular phase. ${ }^{[5]}$

The majority of male infertility (90\%) is due to low sperm number, poor semen quality or a combination of both. Worldwide collected data suggest that there has been a continuous decline in semen quality and quantity. ${ }^{[6]}$ This alarming finding can be attributed to increased prevalence of sexually transmitted diseases (STDs), urogenital infections and modern lifestyle influences. ${ }^{[7]}$ In our population where infertility is considered to be a social stigma and the female is often held responsible for the inability to conceive, screening by semen analysis to rule out the male factor is imperative before subjecting the couple to extensive investigations. ${ }^{[8]}$

In this study we determine the frequency of low sperm count in Indian population and semen parameters include sperm motility and morphological details to identify abnormalities in semen.

\section{Materials and Methods}

The retrospective study was carried out in the Department of Pathology, Government Medical College and Hospital, 
Chandigarh, from June 2013 to June 2016. All these couples were unable to conceive for at least 12 months. All the cases, which included the study, were archived from hospital records.

933 Samples of male partners (933) of women attending the fertility clinic of the hospital between the ages of 20 and 50 years were evaluated. The samples taken were primary infertility cases. Cases of secondary infertility were excluded from the study.

Analysis of semen was performed according to the standard methods outlined by the World Health Organization (WHO laboratory manual for the examination and processing of human semen $5^{\text {th }}$ edition 2010). Parameters outlined included: Appearance: grey/ opalescent; Volume: $2.0 \mathrm{ml}$ or more; $\mathrm{pH}$ : alkaline i.e. 7.2-7.8; Sperm concentration: $>15 \times 10^{6}$ spermatozoa/ $\mathrm{ml}$; Total sperm count: $39 \times 10^{6}$ per ejaculate or more; Motility: $40 \%$ or more including progressive and non progressive motility; Morphology: $4 \%$ or more with normal forms; Viability: 58\% live spermatozoa; White cell count: $<1 \times 10^{9} / \mathrm{ml}$.

Complete sample collection and analysis was done by the same lab technician to avoid inter-laboratory variation. Within 60 minutes of collection, semen analysis was performed and parameters included appearance, morphology, motility, volume, liquefaction, $\mathrm{pH}$, concentration, viability and the occurrence of pus cells. Disposable pipette (graduated) were used to measure semen volume; $\mathrm{pH}$ test was done with the help of $\mathrm{pH}$ paper. After liquefaction, sperm motility was assessed by microscopic evaluation of 200 spermatozoa from different fields. Counting of spermatozoa was done using improved Neubauer's chamber. Viability was assessed with eosin stain. The semen samples were categorized on the basis of sperm count $/ \mathrm{mL}$ of semen in accordance with WHO normal and pathological ranges i.e. normozoospermia (normal sperm count), oligozoospermia, (total number (or concentration, depending on outcome reported) of spermatozoa below the lower reference limit) and azoospermia (no spermatozoa in the ejaculate). The different samples were categorized and compared for ejaculated volume, sperm count, viability, pus cells, motility and morphology.

The following operational definitions were used: Normozoospermia: Sperm count 15 million/ml to 150 million/ml; Oligozoospermia: Sperm count below 15 million/ml; Azoospermia: Complete absence of spermatozoa in the ejaculation; Asthenozoospermia: Reduced sperm motility below the lower reference limit; Teratozoospermia: Abnormal sperm morphology;
Oligoasthenoteratozoospermia: All sperm variables abnormal; Hypospermia: Volume $<2 \mathrm{ml}$; Normospermia: Volume 2-5 ml; and Hyperspermia: Volume $>5 \mathrm{ml}$.

The data was analysed using SPSS software (version 15). Mean \pm Standard deviation (SD) were calculated for sperm count, volume, motility, morphology and pus cells; 95\% confidence interval was calculated for proportions and for means. Mean values were also compared for statistical significance using t-value with level of significance $<0.05$ ( $\mathrm{p}$ value).

\section{Result}

A total 933 semen analysis reports of male partners of infertile couples were analyzed over a period of 3 years. Among the 933 males, the mean age was $30.02 \pm 4.72$ years. Using WHO standard for semen normality, 933 semen samples were analysed, out of these $659(70.6 \%)$ had normozoospermia, 170 (18.2\%) had oligozoospermia and 104 (11.1\%) azoospermia, as depicted in Table 1. On the basis of semen volume, samples were categorized as normospermia $(2-5 \mathrm{ml})$, hypospermia $(<2 \mathrm{ml})$, hyperspermia $(>5 \mathrm{ml})$. The distribution of semen volume is shown in Table 2.

After excluding 104 samples with azoospermia, semen parameters were compared in oligozoospermic and normozoospermic samples for count/sperm concentration (million/ml), volume (ml), liquefaction time (min), viability (\%), motile sperms (including progressive motile and non-progressive motile sperms), immotile sperms, morphologically normal sperms and abnormal sperms (including head, neck and tail abnormalities) and pus cell (per HPF). The oligozoospermic samples had significantly higher percentage of immotile sperms 55.79 \pm 28.00 and abnormal morphology $23.64 \pm 27.28$ compared to normozoospermia in which non-motile sperms were $33.32 \pm 19.40$, and abnormal morphology was $11.45 \pm 9.74$ respectively $(\mathrm{p}<0.001)$.

Comparison of volume showed mean volume of $2.64 \pm 1.38 \mathrm{ml}$ in normozoospermia vs $2.25 \pm 1.17 \mathrm{ml}$ in oligozoospermia ( $\mathrm{p} 0.002$ ), and pus cells $4.33 \pm 4.38 / \mathrm{HPF}$ in normozoospermia vs $5.71 \pm 6.56 / \mathrm{HPF}$ in oligozoospermia. This was statistically significant (p 0.009) (Table 3). Normal motility was observed in $66.66 \pm 19.99$ of normozoospermic vs $44.38 \pm 28.20$ of oligozoospermic samples, and normal morphology of sperms was observed in 88.54 \pm 9.74 of normozoospermic vs $76.43 \pm 27.27$ of oligozoospermic samples $(\mathrm{p}<0.001)$.

Comparison of sperm viability showed mean viability of $69.87 \pm 26.69$ in normozoospermia vs $51.78 \pm 24.15$ in oligozoospermia $(p<0.001)$ and liquefaction time 
$42.76 \pm 10.20 \mathrm{~min}$ in normozoospermia vs $44.25 \pm 10.67 \mathrm{~min}$ in oligozoospermia. This was not statistically significant $(\mathrm{p}$ 0.093) (Table 3).
The proportion of multiple factor abnormalities defects were seen in 253 cases out of 829 cases of both normozoospermia and oligozoospermia as given in Table 4.

Table 1: Frequency of sperm concentration

\begin{tabular}{|c|c|c|}
\hline Category & Frequency (N=933) & Percentage (\%) \\
\hline Normozoospermia & 659 & 70.6 \\
\hline Oligozoospermia & 170 & 18.2 \\
\hline Azoospermia & 104 & 11.1 \\
\hline
\end{tabular}

Table 2: Distribution of semen volume

\begin{tabular}{|c|c|c|}
\hline Volume & Frequency (N=933) & Percentage (\%) \\
\hline Normospermia $(2-5 \mathrm{ml})$ & 658 & 70.5 \\
\hline Hypospermia $(<2 \mathrm{ml})$ & 252 & 27.0 \\
\hline Hyperspermia $(>5 \mathrm{ml})$ & 23 & 2.4 \\
\hline
\end{tabular}

Table 3: Comparison of semen parameters between normozoo-spermia and oligozoo-spermia

\begin{tabular}{|c|c|c|c|c|c|c|c|c|}
\hline Category & $\begin{array}{c}\text { Count } \\
\text { mean } \pm \text { SD }\end{array}$ & $\begin{array}{c}\text { Volume } \\
\text { mean } \pm \text { SD }\end{array}$ & $\begin{array}{c}\text { Viability } \\
\text { mean } \pm \text { SD }\end{array}$ & $\begin{array}{c}\text { Pus cells } \\
\text { mean } \pm \text { SD } \\
\text { median } \pm \text { IR }\end{array}$ & $\begin{array}{c}\text { Motile sperm } \\
\text { mean } \pm \text { SD }\end{array}$ & $\begin{array}{c}\text { Immotile } \\
\text { sperm } \\
\text { mean } \pm \text { SD }\end{array}$ & $\begin{array}{c}\text { Normal } \\
\text { sperm } \\
\text { mean } \pm \text { SD }\end{array}$ & $\begin{array}{c}\text { Abnormal } \\
\text { sperm } \\
\text { mean } \pm \text { SD }\end{array}$ \\
\hline $\begin{array}{c}\text { Normozoo } \\
\text {-spermia }\end{array}$ & $84.98 \pm 32.87$ & $2.64 \pm 1.38$ & $69.87 \pm 26.69$ & $4.33 \pm 4.38$ & $66.66 \pm 19.99$ & $33.32 \pm 19.40$ & $88.54 \pm 9.74$ & $11.45 \pm 9.74$ \\
\hline $\mathbf{9 5 \% C I}$ & $82.46-87.49$ & $2.53 \pm 2.74$ & $67.83-71.91$ & $4.00-4.67$ & $65.13-68.19$ & $31.84 \pm 34.80$ & $87.79-89.28$ & $10.71 \pm 12.20$ \\
\hline $\begin{array}{c}\text { Oligozoo- } \\
\text { spermia }\end{array}$ & $9.78 \pm 3.82$ & $2.25 \pm 1.17$ & $51.78 \pm 24.15$ & $5.71 \pm 6.56$ & $44.38 \pm 28.20$ & $55.79 \pm 28.00$ & $76.43 \pm 27.27$ & $23.64 \pm 27.28$ \\
\hline 95\%CI & $9.20-10.36$ & $2.08 \pm 2.43$ & $48.12-55.44$ & $4.72-6.71$ & $40.11-48.65$ & $51.55 \pm 60.03$ & $72.29-80.55$ & $19.50 \pm 27.77$ \\
\hline P value & $<0.001$ & 0.002 & $<0.001$ & 0.009 & $<0.001$ & $<0.001$ & $<0.001$ & $<0.001$ \\
\hline
\end{tabular}

Table 4: Proportion of multiple factor abnormalities defect

\begin{tabular}{|c|c|c|}
\hline Pattern of abnormalities & Frequency & Percentage (\%) \\
\hline Asthenozoospermia & 118 & 2.9 \\
\hline Teratozoospermia & 24 & 1.39 \\
\hline Asthenoteratozoospermia & 13 & 8.2 \\
\hline Oligoasthenozoospermia & 68 & 2.3 \\
\hline Oligoteratozoospermia & 19 & 1.3 \\
\hline
\end{tabular}

\section{Discussion}

Infertility has long been a subject of debate and the females have always had to bear the brunt of the sociocultural connotations of this multifaceted issue. ${ }^{[1,10]}$ Advancements and progress of novel assisted reproductive techniques establish males to be an equal, if not higher contributor to this complex problem. ${ }^{[2]}$ Despite education and enlightenment, the social attitude towards infertility results in much trauma, emotional instability and psychological stress, which in turn has an adverse bearing on the physiology and psychology of the individual, particularly in a social set-up such as ours, where there has been a strong emphasis on child-bearing. ${ }^{[11]}$ Semen analysis provides some insight about the pathology of epidemiological problems occurring in the male genital tract. ${ }^{[1,4]}$ As high as majority $(90 \%)$ of male infertility problems are connected to sperm count and a positive association between abnormal semen parameters and sperm number, has been observed. The problem of sperm count, motility and morphology stems from disarray in 
control mechanisms, including pre-testicular, testicular and post-testicular factors. ${ }^{[12]}$

In our study it was found that of total 933 cases 659 $(70.6 \%)$ males had normal sperm count and rest 274 (29.3\%) males had abnormal semen analysis report. This is similar to a study done in 2012 which reported the incidence of male infertility as $62 \% .{ }^{[7]}$ The reported prevalence of oligozoospermic, azoospermic, asthenozoospermic and asthenoteratozoospermic in cases of primary infertility in same study was $33.17 \%, 9.89 \%$, 1.83 and $1.08 \%$ respectively, ${ }^{[13]}$ which were similar to our study results. The prevalence of azoospermia in our study population was $10.70 \%$, oligozoospermia $34.14 \%$, asthenozoospemia $14.2 \%$, and of asthenoteratozoospermia $1.39 \%$ respectively. ${ }^{\left[{ }^{[7]}\right.}$ The results are comparable to study which reported the occurrence of azoospermia as $14.28 \%$ and that of oligozoospermia $21.43 \%,{ }^{[14]}$ in another study, the incidence rate of azoospermia was $16 \%{ }^{[15]}$

Mean ejaculated volume in normozoospermia was $2.64 \pm 1.38 \mathrm{ml}$ vs $2.25 \pm 1.17 \mathrm{ml}$ in oligozoospermia and $2.20 \pm 1.30 \mathrm{ml}$ in azoospermic samples respectively. Majority of our patients had normal semen volume $70.5 \%$, while $27.0 \%$ showed hypospermia $(<2 \mathrm{ml})$, and hyperspermia in $2.4 \%$, these results can be comparable to a study conducted in Sudan where majority of the subjects $(89.7 \%)$ had adequate semen volume, while only $10.3 \%$ had abnormal semen volume. ${ }^{[16]}$ Moreover, these results are also analogous to a study conducted in Nigeria in which majority of the subjects $(91 \%)$ had adequate semen volume, while only $9 \%$ had abnormal semen volume i.e 7.3\% hypospermia and $1.7 \%$ hyperspermia. ${ }^{[17]}$ The adequate semen volume obtained in our study may be a result of the 3-6 days of sexual abstinence.

In normozoospermia samples, the mean percentage of normal motile sperms was $57 \% \pm 0.18$ as compared to oligozoospermia in which motile sperms were $38 \% \pm 23 \%$. However, advancing techniques to some extent overcome the problems of sperm motility in infertile couples, but asthenozoospermia is still a common cause of human male infertility. In our study, asthenozoospermia was observed in $14.2 \%$ of samples and the results were comparable to a study conducted at the National Institute of Heath, Islamabad, in which the prevalence was around $21.42 \%$. ${ }^{[18]}$ In another study, the prevalence of asthenozoospermia was $18 \%{ }^{[19]}$

Morphology of a sperm i.e. the differential development of the head, mid-piece and tail is a function of testes as well as the epididymis. In this study in normozoospermia samples, mean normal morphology was found to be $88.54 \pm 9.74 \%$ vs. $76.43 \pm 27.27 \%$ in oligozoospermic samples. The oligozoospermic samples had significantly higher abnormal motility $55.79 \pm 28.00 \%$ and abnormal morphology $23.64 \pm 27.28 \%$ as compared to normozoospermic samples with $33.32 \pm 19.40 \%$ abnormal motility and $11.45 \pm 9.74 \%$ abnormal morphology. These results are comparable to a study in which abnormal morphology was observed in $53 \%$ and abnormal motility in $60 \%$ oligozoospermic males. ${ }^{[20]}$ So sperm motility and morphology are changing parameters and their relative levels depend on the existing sperm count in an individual. ${ }^{[20]}$

Oligozoospermic samples were found to be associated with significant higher abnormal motility $62 \% \pm 0.239$ and abnormal morphology $55 \% \pm 0.156$ as compared to normozoospermic samples although we did not specify the type of abnormal morphology. The results are comparable to a study in which abnormal morphology was observed in $53 \%$ and abnormal motility in $60 \%$ oligozoospermic males. So sperm motility and morphology are changing parameters and their relative levels depend on the existing sperm count in an individual. ${ }^{20]}$

The prognosis of the infertile couple is inversely proportional to the number of abnormal patterns so one pattern of abnormality is better than two-pattern abnormality, and two is better than three-factor abnormality. ${ }^{[21,22]}$ When three-pattern abnormalities were identified in oligozoospermic sample population, the prevalence of oligoasthenoteratozoospermia was $1.3 \%$. The results were comparable to a study in which prevalence of oligoasthenoteratozoospermia was $11 \%$. ${ }^{[18]}$ The prevalence of teratozoospermia in our study population was $2.9 \%$.

\section{Conclusion}

Semen analysis is primary tool to investigate male infertility which is more useful in developing countries like India. It comes under basic investigation done at minimal rates for infertility cases. It is a cost effective, more reproducible and gives robust information of male reproductive function. The use of conventional parameters, such as sperm count, viability, sperm morphology and motility are markers of male reproductive function. Thus, semen analysis serves as a preliminary investigation to rule out male cause of infertility.

\section{Acknowledgements}

We are thankful to all the members of hematology section, Department of Pathology for their great help throughout this work.

\section{Competing Interests}

There are no conflicts of interest. 


\section{Reference}

1. Milardi D, Grande G, Sacchini D, Astorri AL, Pompa G, Giampietro A et al. Male fertility and reduction in semen parameters: a single tertiary-care center experience. Int $\mathrm{J}$ Endocrinol 2012; 649(1155): 149-55.

2. Carlsen E, Giwercman A, Keiding N, Skakkebaek NE. Evidence for decreasing quality of semen during past 50 years. BMJ 1992; 305(6854): 609- 13.

3. Fisch H. Declining Worldwide Sperm Counts: Disproving a Myth. Urol Clin N Am 2008; 35(2): 137-46.

4. Butt F, Akram N. Semen analysis parameters: Experiences and insight into male infertility at a tertiary care hospital in Punjab. J Pak Med Assoc 2013; 63(5):558-62.

5. Acacio BD, Gottfried T, Israel R, Sokol RZ. Evaluation of a large cohort of men presenting for a screening semen analysis. Fertil Steril 2000; 73(3): 595- 7.

6. Wong WY, Thomas CMG, Merkus JM, Zielhius GA, Steegers-theunissen RP. Male factor sub fertility: possible causes and impact of nutritional factors. Fertil Steril 2000; 73(5): 435-42.

7. Samal S, Dhadwe K, Gupta U, Gupta NK. Epidemiological Study of Male Infertility. Indian Medical Gazette 2012; 5: $174-80$

8. Kumar N, Choudhari AR, Singh AK' Prevalence of Male Factor Infertility in Last Ten Years at a Rural Tertiary Care Centre of Central India: A Retrospective Analysis . Ind J Obs and Gynaecol Res 2015; 2:132-36.

9. World Health Organization. WHO laboratory manual for the examination and processing of human semen. 5th ed. Geneva: World Health Organization 2010; 223-6.

10. Kumar A. Fertility and in-vitro fertilization in India. Current Science 2004; 86(2): 254-6.

11. Bhaduri (Bhattacharyya) N, Sarkar AP, Dewasi N, Ghosh TK. Abnormalities in semen analysis among male partners of infertile couples: a study in a tertiary care level hospital of
West Bengal, India. Int J Reprod Contracept Obstet Gynecol 2015; 4(1): 100-2

12. Iwamoto T, Nozawa S, Yoshiike M, Namiki M, Koh E, Kanaya $J$ et al. Semen quality of fertile Japanese men: a cross-sectional population-based study of 792 men. Reprod Med Biol 2007;6(1):185-93.

13. Jequier AM. Semen analysis: a new manual and its application to the understanding of semen and its pathology. Asian J Androl 2010;12(1):11-3.

14. Khan AA, Khan FA, Sattar A, Naveed AK, Ahmed M. Azoospermia in clinical practice at Rawalpindi. Pak Armed Forces Med J 1992; 42(12): 93- 95.

15. Subhan F, Tahir F, Ahmad R, Khan ZU. The study of azoospermic patients in relation to their hormonal profile (LH, FSH and Testosterone). Rawal Med J 1995; 22(6): 25-7.

16. Imam MEI, Siuf A, Mansour MM, Khalid KE, Yosif N, Elhasan EM, Miskeen E. Semen Analysis of Infertile Sudanese Males in Gezira State. Central Sudan. SJPH 2009;4(3): 340-4

17. Nwafia WC, Igweh JC, Udebuani IN. Semen analysis of infertile igbo males in enugu, eastern Nigeria. Niger J Physiol Sci 2006; 21(1-2): 67-70.

18. Subhan F, Tahir, F, Alam W, Sultan S, Shahab M. Seminal and hormonal profiles of fertile and subfertile Pakistani men -a study of infertility cases. Pak J Med Res 2000; 39(3): 42-5.

19. Curi SM, Ariagno JI, Chenlo PH, Mendeluk GR, Pugliese MN, Sardisegovia LM, et al. Asthenozoospermia: analysis of a large population. Arch Androl 2003; 49(5): 343-9.

20. Dua AA, Vaidya SR. Sperm motility and morphology as changing parameters linked to sperm count variations. J Postgrad Med 1996; 42(4): 93-6.

21. Jarow JP, Espeland MA, Lipshultz G. Evaluation of the azoospermic patients. J Urol 1989; 142(1): 62-5.

22. Mathews T, Mati JK, Formula JN. A study of infertility in Kenya. Results of investigation of the infertile couples in Nairobi. East Afr Med J 1981; 58(4): 258-67.

*Corresponding author:

Dr Asif Baliyan, 8/30, Saqlane Road, Batla House, Jamia Nagar, New Delhi-25

Phone: +91 8699661122

Email: drasifbaliyan@gmail.com

Date of Submission : 28.04.2017

Date of Acceptance : 27.06.2017

Financial or other Competing Interests: None.
Date of Publication : 26.10.2017 